\section{Relevance of Endoxifen Concentrations: Absence of Evidence Is Not Evidence of Absence}

\section{TO THE EDITOR:}

We read the article by Sanchez-Spitman et $\mathrm{al}^{1}$ reporting on their CYPTAM study about the association between CYP2D6 genotype and clinical outcome in the adjuvant treatment of breast cancer with tamoxifen. We acknowledge that a lack of association was shown and that solely the determination of CYP2D6 genotype has limited relevance for clinical practice in this setting.

However, we do not agree with Sanchez-Spitman et al ${ }^{1}$ on their interpretation of the data on the association between endoxifen concentrations (which is the most relevant tamoxifen metabolite) and recurrence of breast cancer. In our opinion, the authors are jumping to conclusions, because the primary end point of this study was not to investigate the relationship between endoxifen concentrations and clinical outcome. Instead, the study was amended in 2017 to explore endoxifen concentrations in relation to recurrence-free survival and was underpowered to draw solid conclusions on this end point, with a hazard ratio of 2.0 as input into their sample size calculations. This hazard ratio cannot be considered realistic to study the effects of endoxifen concentrations on recurrence-free survival. ${ }^{2}$

The CYPTAM study included 662 patients from 2008 to 2011 . The trial was originally designed to study CYP2D6 genotype and recurrence-free survival. According to the protocol, one serum sample was taken for pharmacokinetic purposes (at a random moment during the first year of treatment), and the authors correlated the measured endoxifen concentrations with outcome retrospectively. In 2011, Madlensky et $\mathrm{al}^{3}$ reported a hazard ratio of 1.4 for patients with endoxifen concentrations below versus above $5.97 \mathrm{ng} / \mathrm{mL}$. Considering this hazard ratio, and the same assumptions as were made in the CYPTAM protocol (2 years of patient inclusion and 2 years of follow-up), a prospectively designed study would have required 276 events in at least 3,150 patients. Importantly, the ratio between patients with endoxifen concentrations below and above the cutoff point (1:4) differs from the ratio between the phenotype groups as assumed in the original sample size calculations in the CYPTAM study (1:1.25). Hence, an even larger sample size would have been required if the correct ratio was taken into account, leading to almost 4,500 patients in the case of 2 years of inclusion and 2 years of follow-up. Sanchez-Spitman et al ${ }^{1}$ do not mention the observed number of events, nor do they present a Kaplan-Meier curve for recurrence-free survival stratified for endoxifen concentration that could provide some insight into this number. However, the wide confidence intervals of the hazard ratios for different risks groups suggest a low number of events. In addition, according to the study protocol, several patients with an intermediate or poor metabolizer phenotype received a tamoxifen dose increment temporarily. It is unclear from the article whether these patients were included in this analysis, but if so, this has confounded the outcome of the study. In addition, the authors do not discuss their conflicting results about endoxifen concentrations and clinical outcome in comparison with previous studies. ${ }^{3-5}$

Moreover, just one serum sample does not reflect systemic exposure throughout the tamoxifen treatment course. For example, use of comedication may change over time and may seriously affect systemic endoxifen concentrations. ${ }^{6}$ It is known that the combination of tamoxifen and strong CYP2D6 inhibitors is still popular among patients with breast cancer. ${ }^{7}$ Therefore, we believe that it is a shortcoming that data on comedication or other factors (temporarily) influencing endoxifen concentrations (eg, low and variable compliance $^{8}$ ) are missing in this analysis.

Sanchez-Spitman et al ${ }^{1}$ conclude their article by stating that "our data do not justify therapeutic drug monitoring based on endoxifen concentrations in patients with breast cancer receiving tamoxifen." However, on the basis of the reflections mentioned previously, this conclusion cannot be drawn from their study, especially in light of the available literature. ${ }^{3-5}$ Instead, we are opting for a large prospective — if possible, randomizedclinical trial to study the value of endoxifen-based therapeutic drug monitoring in tamoxifen treatment. ${ }^{9,10}$

\section{Louwrens Braal, PharmD}

Erasmus University Medical Center, Rotterdam, the Netherlands

\section{Jos H. Beijnen, PhD}

The Netherlands Cancer Institute-Antoni van Leeuwenhoek, Amsterdam, the Netherlands, and Utrecht University, Utrecht, the Netherlands

\section{Stijn L.W. Koolen, PhD, PharmD and Esther Oomen-de Hoop, PhD}

Erasmus University Medical Center, Rotterdam, the Netherlands

\section{Neeltje Steeghs, MD, PhD}

The Netherlands Cancer Institute-Antoni van Leeuwenhoek, Amsterdam, the Netherlands 
Agnes Jager, MD, PhD

Erasmus University Medical Center, Rotterdam, the Netherlands

\section{Alwin D.R. Huitema, PharmD, PhD}

The Netherlands Cancer Institute-Antoni van

Leeuwenhoek, Amsterdam, the Netherlands, and Utrecht University, Utrecht, the Netherlands

\section{Ron H.J. Mathijssen, MD, PhD}

Erasmus University Medical Center, Rotterdam, the Netherlands

\section{AUTHORS' DISCLOSURES OF POTENTIAL CONFLICTS OF INTEREST AND DATA AVAILABILITY STATEMENT}

Disclosures provided by the authors and data availability statement (if applicable) are available with this article at DOI https://doi.org/10.1200/ JCO.19.00418

\section{REFERENCES}

1. Sanchez-Spitman A, Dezentjé V, Swen J, et al: Tamoxifen pharmacogenetics and metabolism: Results from the prospective CYPTAM study. J Clin Oncol 37:636-646, 2019

2. Early Breast Cancer Trialists' Collaborative Group (EBCTCG): Effects of chemotherapy and hormonal therapy for early breast cancer on recurrence and 15-year survival: An overview of the randomised trials. Lancet 365: 1687-1717, 2005

3. Madlensky L, Natarajan L, Tchu S, et al: Tamoxifen metabolite concentrations, CYP2D6 genotype, and breast cancer outcomes. Clin Pharmacol Ther 89:718-725, 2011

4. Saladores P, Mürdter T, Eccles D, et al: Tamoxifen metabolism predicts drug concentrations and outcome in premenopausal patients with early breast cancer. Pharmacogenomics J 15:84-94, 2015

5. Helland T, Henne N, Bifulco E, et al: Serum concentrations of active tamoxifen metabolites predict long-term survival in adjuvantly treated breast cancer patients. Breast Cancer Res 19:125, 2017

6. Stearns V, Johnson MD, Rae JM, et al: Active tamoxifen metabolite plasma concentrations after coadministration of tamoxifen and the selective serotonin reuptake inhibitor paroxetine. J Natl Cancer Inst 95:1758-1764, 2003

7. Binkhorst L, Mathijssen RH, van Herk-Sukel MP, et al: Unjustified prescribing of CYP2D6 inhibiting SSRIs in women treated with tamoxifen. Breast Cancer Res Treat 139:923-929, 2013

8. Hershman DL, Shao T, Kushi LH, et al: Early discontinuation and non-adherence to adjuvant hormonal therapy are associated with increased mortality in women with breast cancer. Breast Cancer Res Treat 126:529-537, 2011

9. Koolen SLW, Bins S, Mathijssen RHJ: Individualized tamoxifen dose escalation-letter. Clin Cancer Res 22:6300, 2016

10. de Vries Schultink AHM, Huitema ADR, Beijnen JH: Therapeutic drug monitoring of endoxifen as an alternative for CYP2D6 genotyping in individualizing tamoxifen therapy. Breast 42:38-40, 2018

DOI: https://doi.org/10.1200/JC0.19.00418; Published at jco.org on June 18, 2019. 


\section{Relevance of Endoxifen Concentrations: Absence of Evidence Is Not Evidence of Absence}

The following represents disclosure information provided by authors of this manuscript. All relationships are considered compensated. Relationships are self-held unless noted. I = Immediate Family Member, Inst = My Institution. Relationships may not relate to the subject matter of this manuscript. For more information about ASCO's conflict of interest policy, please refer to www.asco.org/rwc or ascopubs.org/jco/site/ifc.

\section{Jos H. Beijnen}

Employment: Modra Pharmaceuticals

Stock and Other Ownership Interests: Modra Pharmaceuticals

Honoraria: Modra Pharmaceuticals

Research Funding: Roche, PharmaMar, Neon Therapeutics, Astex

Pharmaceuticals, Tesaro

Patents, Royalties, Other Intellectual Property: Patent dealing with the pharmaceutical formulation of oral taxanes (Inst)

\section{Stijn L.W. Koolen}

Speakers' Bureau: Novartis, Pfizer

Research Funding: Cristal Therapeutics (Inst), Novartis (Inst)

Travel, Accommodations, Expenses: Ipsen

\section{Neeltje Steeghs}

Research Funding: AstraZeneca/Medlmmune (Inst), Bayer (Inst), Bristol-Myers Squibb (Inst), Novartis (Inst), GlaxoSmithKline (Inst), Pfizer (Inst), Roche (Inst), Genentech (Inst), Boehringer Ingelheim (Inst), Blueprint Medicines (Inst), AB Science (Inst), Deciphera (Inst), Genentech (Inst), Merck Sharp \& Dohme (Inst), Amgen (Inst), Merus (Inst), Lilly (Inst), Incyte (Inst)

\section{Ron H.J. Mathijssen}

Speakers' Bureau: Novartis

Research Funding: Roche (Inst), Sanofi (Inst), Astellas Pharma (Inst), Bayer Holding (Inst), Boehringer Ingelheim (Inst), Pfizer (Inst), Cristal Therapeutics (Inst), Pamgene (Inst)

Travel, Accommodations, Expenses: Pfizer, Astellas Pharma

No other potential conflicts of interest were reported. 